\title{
The Food Systems Dashboard is a new tool to inform better food policy
}

The Food Systems Dashboard brings together extant data from public and private sources to help decision makers understand their food systems, identify their levers of change and decide which ones need to be pulled.

\author{
Jessica Fanzo, Lawrence Haddad, Rebecca McLaren, Quinn Marshall, Claire Davis, Anna Herforth, \\ Andrew Jones, Ty Beal, David Tschirley, Alexandra Bellows, Lais Miachon, Yuxuan Gu, Martin Bloem and \\ Arun Kapuria
}

$\mathrm{T}$ he Global Burden of Disease study showed that unhealthy diets contribute to 11 million deaths per year ${ }^{1}$. The double burden of malnutrition - the coexistence of overweight, obesity and non-communicable diseases with underweight, micronutrient deficiencies, wasting and stunting - is being driven by changes in food systems and in some cases increased availability of cheap, highly processed, nutrient-poor foods, impacting the lowest-income countries in sub-Saharan Africa, South and East Asia, and the Pacific the hardest ${ }^{2}$

Diets are shaped by food systems. Food systems are made up of all the people, institutions, environments, infrastructure and activities that relate to the production, processing, distribution, marketing, sale, preparation and consumption of food ${ }^{3}$. Food systems are intrinsically related to health, environment, culture, politics and economy. The food systems framework depicts these outcomes as well as characteristics such as food availability and affordability and personal knowledge, preferences, resources and behaviours (Fig. 1). Policy interventions that address one part of the system will impact many outcomes that food systems contribute to ${ }^{4}$. Importantly, actions can have both unintended consequences and multiple benefits due to this interconnectivity ${ }^{5}$.
Though there is widespread agreement that our food systems are unsustainable ${ }^{6,7}$, identifying ways to change and improve them is difficult. Food systems are complex and offer many entry points for change ${ }^{8}$. Additionally, even when actions have been identified, they often lack public acceptance and may not be politically feasible.

However, it has been found that policies can be modified or combined in ways that increase their acceptance and, therefore, policy packaging is an important strategy to make policies both effective and politically feasible ${ }^{9}$. Policymakers, non-governmental organizations, civil society leaders and other actors do not currently have a holistic

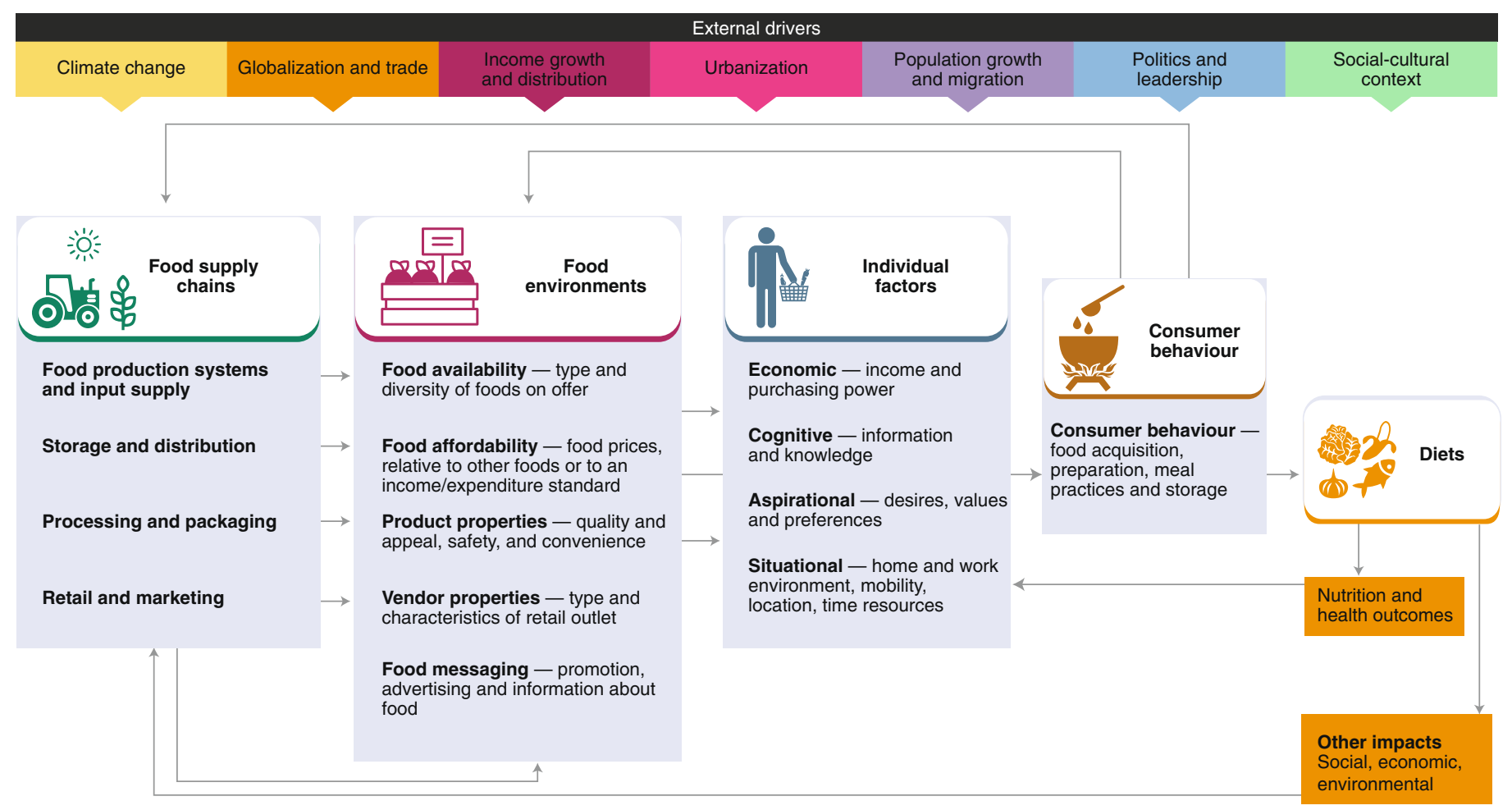

Fig. 1 | Food Systems Framework. The different components of food systems, their drivers and outcomes are interconnected. Figure adapted with permission from ref. "1". 


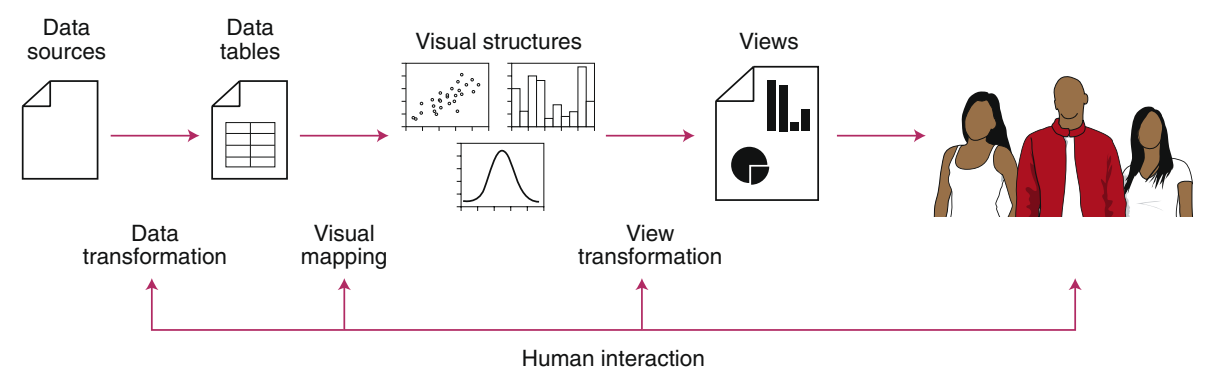

Fig. 2 | The steps involved in shaping the Food Systems Dashboard. Data visualization and transformation leads to dissemination.

tool to enable visualization of their own national food systems, understand the interconnections across multiple sectors, perform comparisons with other countries, identify key challenges and prioritize actions.

This lack of accessible information on the status quo significantly hinders evidence-based policymaking to improve food systems. Given the level of complexity and interconnections inherent to food systems, the data that describe these systems and their linkages to diets and nutrition need to be aggregated and presented in a way that is easily understandable ${ }^{10}$. Data visualizations are potentially an important way to facilitate understanding, decision making and advocacy.

\section{The Food Systems Dashboard}

The Food Systems Dashboard is a new tool that aims to describe global, regional and national food systems; to assess the challenges for improving diets, nutrition and health; and to guide its users to set priorities and decide on actions. The need for this tool was identified by Jess Fanzo at Johns Hopkins University and Lawrence Haddad at The Global Alliance for Improved Nutrition (GAIN) in 2018 when working on the team that wrote the UN High Level Panel of Experts on Food Systems and Nutrition report ${ }^{11}$. Work on the Dashboard started that year, bringing together a team from Johns Hopkins University, GAIN, Harvard University, the University of Michigan and Michigan State University. Once the framework was finalized, the team worked to find indicators that described the different components of food systems and had high-quality data for countries at all income levels. The team started working with iTech Mission in 2019 to create the website and continually improve its design and usability. The Dashboard is still currently in development and the launch of the beta version is forthcoming in June. The data are publicly accessible via the online
Dashboard, which has a well-designed and easy-to-navigate user interface, as designed by iTech Mission with user testing and feedback from our team and additional pilot testing and modifications planned following the launch. Figure 2 shows how food systems data are transformed from original data sources to metadata that can be altered through data structural changes and visual mapping resulting in graphical views of data. iTech has visual information design experience across a range of platforms, including the Sustainable Development Goals (SDGs) Dashboard. Throughout the process of designing the Food Systems Dashboard, we have brought together a diverse set of perspectives, with some more experienced with data navigation and others less so, to ensure that the tool is ready before it reaches decision makers. The next step will be to test with those working in food systems in a diverse set of countries who need to understand the data to make sound decisions.

Describing food systems. The Dashboard describes food systems by bringing together extant data across over 140 indicators from over 30 sources. These sources, which are both public and private, include UN agencies, the World Bank, CGIAR agencies, Euromonitor and cross-country research. The indicators are organized using a conceptual framework adapted from the High-Level Panel of the UN Committee on Food Security in 2017, as shown in Fig. $1^{5}$. The framework describes the entire food system, including food supply chains, food environments, individual factors, consumer behaviour, diets and nutrition, and environmental, social, political and economic drivers - factors that push or pull the system. Aggregating these diverse data will improve stakeholders' understanding of their national food systems in terms of the different food systems' components (food supply chains, food environments and individuals), their cross-sectional nature and how these components may influence diet and nutrition outcomes. The Dashboard will provide country profile snapshots of a curated set of indicators that capture these components in an 'infographic' type visual that explains the data, is easy to understand, shows the connections and can be downloaded for dissemination purposes. The country profiles are meant to tell a story about a country's food system.

Assessing food systems. The Dashboard enables stakeholders to compare their food systems with those of other countries. This comparison can be done regionally, by income classification, or based on a food system typology. To develop the typologies, 146 countries were grouped into five country-level food system types using a composite index score. The typologies are meant to characterize broad patterns across households, neighbourhoods, regions and countries in their agricultural production practices, supply chains and food environments. Though it is recognized that the full complexity of food systems and heterogeneity across countries cannot be adequately conveyed through a typology, typologies may be useful in identifying broad patterns across countries and better enabling countries to learn from one another. The Dashboard has developed case studies of these five food system typologies that provide a typical context of what one may find in these typologies and there are plans to develop more cases that illustrate how food system types are characterized and changing in the context of other macro-drivers such as urbanization and climate change.

Prioritizing actions. The Dashboard will provide guidance on potential priority actions to improve food systems' impacts on diets and nutrition. These actions may take the form of policy and programme interventions, tools, or investments. It proposes the food system actors that need to be involved in making the desired changes. While the current focus is on diets and nutrition, the dashboard includes several environmental and natural resource indicators that are important for the resilience of food systems and these will be expanded to increase the focus on sustainability. The methods to develop priority actions are in progress, but there are several policy streams that will inform the key evidence-based policies that can impact diets and nutrition, including City, University of London's and GAIN's work on No Regrets Policy Actions and the UN Voluntary Guidelines on Food Systems for Nutrition. 
Decision-making. The Dashboard is intended as the primary resource for decision makers to find curated, high-quality data and analytics on their country's food systems. The data gives users insight into the state of their food systems and their effects on nutrition and health. The Dashboard also suggests parts of the food system that may require corrective action through actionable indicators. The Data for Decisions to Expand Nutrition Transformation (DataDENT) Initiative - a four-year initiative led by Johns Hopkins University, the International Food Policy Research Institute and Results for Development aimed at transforming the availability and use of nutrition data found that providing such guidance on needed corrective actions is important to increase the practical utility of data visualization tools such as dashboards ${ }^{12}$. The Food Systems Dashboard will provide broad recommendations for policy, programme and investment actions to address these food systems' shortcomings using actionable indicators. We hope that this will facilitate higher-quality decision-making to construct better National Food System Action Plans, which ultimately will advance human and planetary health.

\section{Advance of the dashboard}

With advanced information connectivity, it is perhaps predictable that a number of data visualization tools are proliferating in the nutrition space. The DataDENT Initiative found that there were over 22 global visualization tools in the nutrition space alone, with at least 14 of these tools launched or refreshed between July 2017 and June $2018^{12}$. The Initiative reported overlap and inconsistencies within available tools, which can cause confusion and fatigue for decision makers ${ }^{12}$. The Dashboard is taking the lessons learned from these tools and working to ensure the data are visually appealing and easy to understand. We will also test the Dashboard with different users to see what types of visual are the most understandable across various stakeholders.

The Dashboard brings together data from many different sources. The data provide insights into different aspects of food systems that are not commonly included in other nutrition data visualization tools, allowing users to understand food systems in a more comprehensive way. In addition to diets, nutrition and health indicators, the Dashboard also includes indicators from other sectors - agriculture, food prices, retail, marketing, climate change, urbanization, poverty, literacy and others - that connect to food systems. The Dashboard presents data on almost all countries, whereas other food indices and platforms often only capture data from countries with many data points. These countries are usually a select number of high-income countries.

\section{Developing the dashboard}

Limited data availability is a challenge that hinders all data visualization tools - the Dashboard included. The DataDENT Initiative showed that in many data visualization tools there are no data for the indicators of interest, data are out of date, trend data are not available, data are only available for a small number of countries, or data are not available at the geographic level needed such as sub-national data ${ }^{12}$. Given these limitations, the Dashboard can also be used as a tool for advocacy. We hope that flagging important indicators that have limited or no data will generate more data collection and sharing. More data are needed, especially for diets, individual factors, consumer behaviour and food environments, as well as disaggregated data at sub-national levels. We are reaching out to research groups around the world from various disciplines and sectors who are willing to work with us and share data.

The dietary data included in the Dashboard are from the Global Burden of Disease study. While these are the most accessible data currently available, they are from modelled estimates that may underor over-represent actual dietary intakes. While there may be typical diets in some countries, there is also a lot of variation and thus individual dietary intake data that are nationally representative are critical, but this does not exist. Thus, data are needed on dietary intakes for people in different age groups, genders and in different regions. The World Bank's Living Standards Measurement Study (LSMS) includes data on food consumption and expenditures, which would be a useful addition to the Dashboard, but these data would need to be processed in a comparable way to the Global Burden of Disease data to be included. Specifically, household expenditures on various items would have to be combined such that expenditures on individual dietary risk factors, including vegetables (as a whole group), legumes, nuts and seeds, sugar-sweetened beverages, processed meats and other food groups, can be determined. Additionally, household expenditures would need to be analysed to determine expenditures on food components that are dietary risk factors, such as expenditures on fibre and sodium.

For individual factors - a person's economic status, knowledge, aspirations and life situation - limited information is available. Of all these, the Dashboard only has robust data for economic factors (that is, income). Data on individual information, knowledge and preferences, as well as food acquisition and consumer behaviour, would further strengthen understanding of the relationship between food systems, behaviour and diets. For example, it would be useful to better use the LSMS and other Household Consumption and Expenditure Surveys (HCES) data to characterize food acquisition at the household level in a centralized data repository. Additionally, while there are data on food sales, that data were determined to be more of a reflection of the food environment and food prices rather than food acquisition. Further, there are limited data available for consumer behaviour related to food preparation, meal practices and storage. These areas of consumer behaviour, more commonly addressed in smaller-scale qualitative studies, are all critical determinants of diets and health, and we urgently need to collect data to better understand them and take informed action.

Food environments also need better and more complete data. While food availability, food prices and access to specific food outlet types (often in urban areas) have been fairly well characterized, we have limited data available on other aspects of the food environment, such as food quality and safety. In particular, food safety indicators are needed to assess contamination of food with toxins, chemical contaminants and adulteration, in addition to statistics on foodborne illness in light of the Ebola epidemic and COVID-19 pandemic. Food marketing and packaging data are also needed to shed light on desirability and convenience of foods - much of these data may be collected but are inaccessible behind paywalls. Another critical gap is the understanding of how individual factors interact with food environment characteristics to determine the desirability and convenience of certain foods ${ }^{13}$.

The Food Systems Dashboard will be launched 1 June 2020 and can be accessed at foodsystemsdashboard.org. The Dashboard will continually be updated with new data, new indicators and sub-national data when available. Over the course of 2020, the Dashboard will be piloted among policymakers in several countries with distinct food system challenges, including Tanzania and Indonesia, as well as others to be determined in the future, to assess usability, utility, and sub-national data collation for deeper food systems analysis.

The Food Systems Dashboard is a community resource that we believe has 
the capacity to allow policymakers to understand their national food systems and the challenges they face, to prioritize and decide on actions to improve diets for health. We are seeking more data from those working in food metrics and databases, more partners and, ultimately, more users to contribute to, and avail of, this resource.

\section{Jessica Fanzo $0^{1,2,3 凶}$}

Lawrence Haddad ${ }^{4}$, Rebecca McLaren', Quinn Marshall ${ }^{3}$, Claire Davis',

Anna Herforth ${ }^{5}$, Andrew Jones ${ }^{6}$, Ty Beal (D) 4 David Tschirley ${ }^{7}$, Alexandra Bellows ${ }^{3}$,

Lais Miachon (D)3 ${ }^{3}$ Yuxuan $\mathrm{Gu}^{3}$, Martin Bloem ${ }^{3}$ and Arun Kapuria ${ }^{8}$

${ }^{1}$ The Berman Institute of Bioethics, Johns Hopkins University, Baltimore, MD, USA. ${ }^{2}$ The Nitze School of Advanced International Studies, Johns Hopkins University, Baltimore, MD, USA. ${ }^{3}$ The Bloomberg
School of Public Health, Johns Hopkins University, Baltimore, MD, USA. ${ }^{4}$ Global Alliance for Improved Nutrition. ${ }^{5}$ Harvard T.H. Chan School of Public Health, Boston, MA, USA. ${ }^{6}$ School of Public Health, University of Michigan, Ann Arbor, MI, USA.

${ }^{7}$ College of Agriculture and Natural Resources, Michigan State University, East Lansing, MI, USA. ${ }^{8}$ iTech Mission, New Delhi, India.

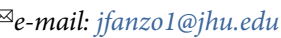

Published online: 13 May 2020

https://doi.org/10.1038/s43016-020-0077-y

References

1. Afshin, A. et al. Lancet 393, 1958-1972 (2019).

2. Popkin, B. M., Corvalan, C. \& Grummer-Strawn, L. M. Lancet 395, 65-74 (2020).

3. Parsons, K. \& Hawkes, C. Connecting Food Systems for Co-benefits: How Can Food Systems Combine Diet-related Health with Environmental and Economic Policy Goals? (City University, London, 2018).

4. Béné, C. et al. Glob. Food Secur. 23, 149-159 (2019).

5. Foran, T. et al. World Develop. 61, 85-101 (2014).

6. Willett, W. et al. Lancet 393, 447-492 (2019).
7. IPCC Climate Change and Land. Special Report on Climate Change, Desertification, Land Degradation, Sustainable Land Management, Food Security, and Greenhouse Gas Fluxes in Terrestrial Ecosystems (eds Shukla, P. R. et al.) (IPCC, 2019).

8. Béné, C. et al. Sci. Data 6, 279 (2019).

9. Fesenfeld, L. P., Wicki, M., Sun, Y. \& Bernauer, T. Nat. Food 1, 173-182 (2020).

10. Global Panel Improved Metrics and Data are Needed for Effective Food System Policies in the Post-2015 Era (Global Panel on Agriculture and Food Systems for Nutrition, 2015).

11. HLPE Nutrition and Food Systems. A Report by the High Level Panel of Experts on Food Security and Nutrition of the Committee on World Food Security (FAO, Rome, 2017).

12. Manorat, R., Becker, L. \& Flory, A. Sight Life 33, 108-114 (2019). 13. Turner, C. et al. Adv. Nutr. 11, 387-397 (2020).

Acknowledgements

The development of the Food Systems Dashboard has been supported by the Johns Hopkins University's Alliance for a Healthier World, The Bill and Melinda Gates Foundation, German Federal Ministry of Economic Cooperation and Development (BMZ), Irish Aid, Ministry of Foreign Affairs, Netherlands, IDRC, and Swiss Development Corporation.

Competing interests

The authors declare no competing interests. 